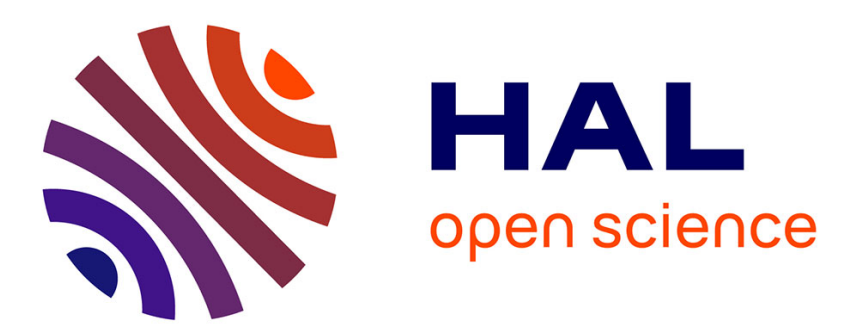

\title{
Similar Triangles and Orientation in Plane Elementary Geometry for Coq-based Proofs
}

Tuan Minh Pham

\section{To cite this version:}

Tuan Minh Pham. Similar Triangles and Orientation in Plane Elementary Geometry for Coq-based Proofs. SAC 2010 - 25th ACM International Symposium on Applied Computing, Mar 2010, Sierre, Switzerland. 10.1145/1774088.1774358 . inria-00585203

\section{HAL Id: inria-00585203 https://hal.inria.fr/inria-00585203}

Submitted on 12 Apr 2011

HAL is a multi-disciplinary open access archive for the deposit and dissemination of scientific research documents, whether they are published or not. The documents may come from teaching and research institutions in France or abroad, or from public or private research centers.
L'archive ouverte pluridisciplinaire HAL, est destinée au dépôt et à la diffusion de documents scientifiques de niveau recherche, publiés ou non, émanant des établissements d'enseignement et de recherche français ou étrangers, des laboratoires publics ou privés. 


\title{
Similar Triangles and Orientation in Plane Elementary Geometry for Coq-based Proofs
}

\author{
Tuan Minh PHAM \\ INRIA Sophia Antipolis - Méditerranée \\ tuan-minh.pham@sophia.inria.fr
}

\begin{abstract}
In plane elementary geometry, the concept of similar triangles not only forms an important foundation for trigonometry, but it also can be used to solve many geometric problems. The notion of orientation allows us to remove the usual ambiguities in presentation of object. In this paper, we present the formalization of these notions in Coq. We also introduce their properties and how they are applied to the proof of two theorems: the Ptolemy's theorem and the Intersecting Chords theorem.
\end{abstract}

\section{Categories and Subject Descriptors}

\section{I.2.3 [ Deduction and Theorem Proving]}

\section{General Terms}

Theory

\section{Keywords}

geometric theorem proving, orientation, similar triangles, formalization, Coq

\section{INTRODUCTION}

Formalizing mathematics allows to verify machenically all of the steps of proofs by a proof assistant system. For elementary geometry, it is more important by the fact that correctness of a traditional proof is affected by the exactness of figures.

In the Coq, some approaches are based on the axiom systems of Hilbert, Tarski [3, 5] to formalize geometric constructions. For formalizing proofs, we can cite here work using the area method [5]. This method reduces many concepts to a single one, and thus somtimes obfuscates the reasoning process.

Another interesting formalization of F.Guilhot [1] for high school curriculum. Its formalized proofs are the traditional ones. However, it lacks many notions. In this context, we

Permission to make digital or hard copies of all or part of this work for personal or classroom use is granted without fee provided that copies are not made or distributed for profit or commercial advantage and that copies bear this notice and the full citation on the first page. To copy otherwise, to republish, to post on servers or to redistribute to lists, requires prior specific permission and/or a fee.

SAC'10 March 22-26, 2010, Sierre, Switzerland.

Copyright 2010 ACM 978-1-60558-638-0/10/03 ...\$10.00. chose the notion of similar triangles and the notion of orientation to enrich it, we also deal with application of these notions in proof of two in the top hundred list [4], being the Ptolemy's theorem and the Product of Segments of Chords

\section{SIMILAR TRIANGLES}

Two triangles are said to be similar (denoted by $\simeq$ ) when all corresponding angles are equal. We separate similarity into 2 types (direct and inverse) by the rotational sense.

\subsection{Properties}

A triangle and its image after an Euclidean transformations are congruent triangles (denoted by $\simeq$ ), which are a special case of similar triangles. Thanks to these properties, we have the proportionality in similar triangles.

Lemma SimTriangles_Proportion : forall $A B C A^{\prime} B^{\prime} C^{\prime}$ $: P O, A B C \sim A^{\prime} B^{\prime} C^{\prime} \rightarrow \frac{A B}{M N}=\frac{B C}{N P}=\frac{C A}{P M}$

To prove this property, the case of inverse similarity is reduced to the one of direct similarity thanks to a reflection, so we continue in the last case. triangle $A B$ " $C$ " is the image of $\triangle M N P$ after applying a rotation to have $M N \| A B$ and a translation to have $M \equiv A$. So we have $\triangle M N P \simeq$ $\triangle A B " C$ " and we can prove that $B C \| B " C ", \mathrm{~B} "$ lies on AB, C" lies on AC. The configuration of these point satisfies Thales' theorem proved in Coq. So we have $\frac{A B}{A B^{\prime \prime}}=\frac{B C}{B^{\prime \prime} C^{\prime \prime}}=$

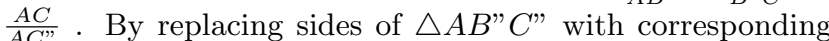
ones of $\triangle M N P$, we get $\frac{A B}{M N}=\frac{B C}{N P}=\frac{C A}{P M}$.

\section{ORIENTATION}

We chose their enumerated order in counter-clockwise direction to talk about orientation (denoted by $\circlearrowleft$ ). We define it by the positive value of the signed area. Definition $\circlearrowleft A B C:=|\overrightarrow{A B}| *|\overrightarrow{A C}| * \sin (\overrightarrow{A B} \overrightarrow{A C})$.

\subsection{Properties}

The first interesting property is about the equality of 2 inscribed angles which intercept the same arc and have the same orientation.

Lemma InscirbedAngles_orient_equal:

forall (OA B CD:PO) ( $r: R)$, concyclic $O r A B C D \rightarrow$ $\circlearrowleft A C D \rightarrow \circlearrowleft B C D \rightarrow \overrightarrow{A C} \overrightarrow{A D}=\overrightarrow{B C} \overrightarrow{B D}$

The second one relating to relative positions of points, obtained after verifying all properties given in $[2,3]$.

Lemma Exists Intersection:

forall ( $O A B C: P O)$, vecBetween2Vecs $O A B C \rightarrow$ 


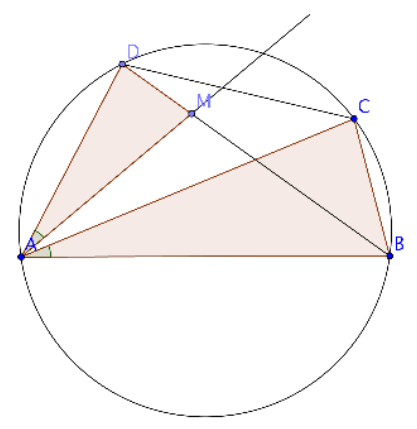

Figure 1: Demonstration of Ptolemy's theorem

exists $E: P O$, isInSegment $A B E \wedge$ isSameSide $O C E$.

Where vecBetween 2Vec $O A B C$ describes that $\overrightarrow{O C}$ is between $\overrightarrow{O A}$ and $\overrightarrow{O B}$, it is defined by $\circlearrowleft O A \wedge \circlearrowleft O C B \wedge$ $\circlearrowleft O A B$, The notion isSameSide $P_{0} P_{1} P_{2}$ describes that $P_{2}$ is in the same side with $P_{1}$ with respect to $P_{0}$.

\section{SOME APPLICATION}

\subsection{Ptolemy's Theorem}

Let a convex quadrilateral ABCD be inscribed in a circle, Ptolemy's theorem is stated by $A D * B C+A B * C D=$ $A C * B D$.

Theorem Ptolemy: forall ( $O A B C D: P O)(r: R)$, convexQuad $A B C D \rightarrow$ concyclic $O$ r $A B C D \rightarrow$ $A D * B C+A B * C D=A C * B D$.

Where the convex quadrilateral is defined by using orientation.

Definition convexQuad $(A B C D: P O):=\circlearrowleft A B C \wedge$

$\circlearrowleft B C D \wedge \circlearrowleft C D A \bigwedge \circlearrowleft D A B$

The traditional proof of this theorem is realized by locating a point $\mathrm{M}$ in the segment $\mathrm{BD}$ such that $\overrightarrow{A B} \overrightarrow{A C}=$ $\overrightarrow{A M} \overrightarrow{A D}$ (see figure 1). With $\triangle A B C \sim \triangle A M D$, we have $B C * A D=M D * A C(1)$. With $\triangle A C D \sim \triangle A B M$, we have $A B * C D=B M * A C(2)$. The addition of (1)(2) give us $A B * C D+B C * A D=(B M+M D) * A C(3)$. By the fact that $\mathrm{M}$ is in the segment $\mathrm{BC}$, which means $B M+M D=B D(4)$, we get the result.

The most important of this proof is (4). To have it, we need to prove the existence of $\mathrm{M}$ in the segment BD. By the definition of convexQuad $A B C D$, we have that $\overrightarrow{A C}$ is between $\overrightarrow{A B}$ and $\overrightarrow{A D}$, so $\overrightarrow{A M}$ is between $\overrightarrow{A B}$ and $\overrightarrow{A D}$. By applying the lemma Exists_Intersection with $\overrightarrow{A M}, \overrightarrow{A B}, \overrightarrow{A D}$, we get that $\mathrm{M}$ in the segment BD. (Q.e.d.)

\subsection{Product of Segments of Chords}

This theorem states that if two chords $\mathrm{AB}$ and $\mathrm{CD}$ intersect at a interior point $\mathrm{M}$ of a circle, so we have $M A * M B=$ $M C * M D$.

Theorem Chords: forall ( $O A B C D M: P O)(r: R)$, concyclic $O$ r A B CD $\rightarrow$ insideCircle $O r M \rightarrow$ liesOn $A B$ $M \rightarrow$ liesOn $C D M \rightarrow M A * M B=M C * M D$.

Where insideCircle $O r M:=$ exists $I J: P O$, onCircle $I O$ $r \wedge$ onCircle $J$ O $r \wedge$ isInSegment I $J M$.

The proof is simple, it uses the proportionality in similar triangles $\triangle M A C \sim \triangle M D B$.

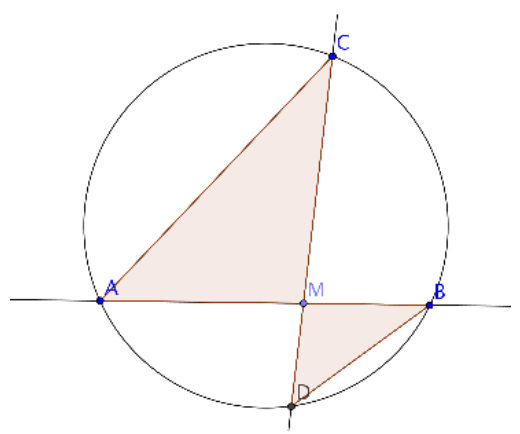

Figure 2: Product of Segments of Chords

However, to obtain this similarity by using the equality of inscribed angles, we need to have that $\mathrm{M}$ is interior to the both segments $\mathrm{AB}$ and $\mathrm{CD}$. We consider the following property which is a consequence of lemma Exists_Intersection for the case of 4 concyclic points.

Lemma Exists_Intersection_Concyclic:

forall ( $A B I J M O: P O)(r: R)$, concyclic $O r A B I J$ $\rightarrow$ isInSegment $I J M \rightarrow$ liesOn $A B M \rightarrow$ isInSegment $A$ $B M$.

By the definition of insideCircle $O r M$, we have 2 point $\mathrm{I}, \mathrm{J}$ on the circle and $\mathrm{M}$ lies in this segment. By applying the property Exists_Intersection_Concyclic with the pair IJ AB and the pair IJ CD, we get that $\mathrm{M}$ is interior to the both segments $\mathrm{AB}$ and CD. (Q.e.d.)

\section{CONCLUSION}

Developing a library to support the proof of geometric theorems in Coq for High School is useful. It helps us verify correctness of traditional proofs. The implementation in more 2000 code lines in Coq system with about 30 lemmas and theorems. New notions were formalized and their properties were verified, enough to prove 2 famous theorems which hadn't been formalized in Coq before.

A lot of work still remains to be done to verify if our notions are well formalized, specifically the notion of orientation. Moreover, there are still many notions not formalized in our library.

This work is only the first step in constructing an interactive proof system. We would like to build a system where each step in a drawing figure is translated into statement or a proof step. A proof library in Coq would be used as backend to verify steps and give us useful results or suggestions.

\section{REFERENCES}

[1] F. Guilhot. Formalisation en Coq et visualisation d'un cours de géométrie pour le lycée. Technique et Science Informatiques, vol 24, p 1113-1138, Hermes Science, 2005.

[2] D. Knuth. Axioms and Hulls. Lecture Notes in Computer Science, vol 606, Springer-Verlag, 1991.

[3] J. Duprat. Une axiomatique de la géométrie plane en Coq. In Actes des JFLA 2008, p 123-136, INRIA, 2008.

[4] Formalizing 100 Theorems http://www.cs.ru.nl/ freek/100/

[5] J.Narboux,Formalisation et automatisation du raisonnement géométrique en Coq. Phd thesis, Paris, 2006 